$$
\begin{aligned}
\text { NAI PROPOSAL No. } 108 \\
\text { Correspondent: } \\
\begin{array}{l}
\text { M. Awschalom } \\
\text { Radiation Physics } \\
\text { National Accelerator Lab } \\
\text { Batavia, Ill. 60510 }
\end{array} \\
\text { FTS/Commercial } 312-231660 \text { Ext } 461
\end{aligned}
$$

\title{
A BEAM DUMP EXPERIMENT
}

M. Awschalom, P. Gollon, F. Schamber

D. Theriot, T. Toohig, A. VanGinneken 


\section{A BEAM DUMP EXPERIMENT}

\section{$\underline{\text { ABSTRACT }}$}

A series of related experiments are proposed to gather data relevant to the understanding of the accelerator operation and planning of new experimental devices and facilities from the point of view of radiation effects.

The data sought are,

1. spatial distribution of density of energy deposition,

2. spatial distribution of radioactivation of steel, concrete and selected soil and water specimens,

3. hadron cascade development,

4. undifferentiated total hadron yields at large angles,

5. muon production and attenuation characteristics, all of them in a massive ("thick") shield such as a beam stop.

EXPERIMENTERS
M. Awschalom
F. Schamber
T. Toohig
P. Gollon
D. Theriot
A. VanGinneken

DATE January 13, 1971

CORRESPONDENT

M. Awschalom

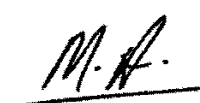

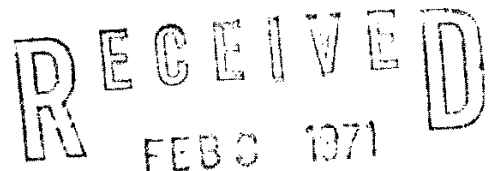

NAL E*osors Onis 


\section{A BEAM DUMP EXPERIMENT}

NAL Radiation Physics Group in

Collaboration with Experimental Facilities*

\section{A. Justification}

The objective of this experiment is to gather information on extranuclear cascades due to very high energy protons incident on steel. This will provide very much needed background data for the design of experimental beams, targets, dumps and future accelerator components, and the understanding of the operation of the accelerator from the point of view of radiation effects. They will also aid in extrapolating to greater beam powers with more confidence from the points of view of personnel and environmental radiation protection.

The results will be compared with existing Monte carlo calculations using various hadron yield formulae (CKP, Trilling, Wang, Hagedorn-Ranft, etc.).

In particular the study of the density of energy deposition by high energy protons in various materials is needed to design dumps regarding optimum dimensions, thermal stresses, cooling, etc. In the future, problems in building aumps will be faced often and optimum design will become important as accelerator current and energy will increase.

* Correspondent: M. Awschalom 
This experiment is in a sense a continuation of a series of similar studies at lower energies performed by several groups which were mainly concerned with the extranuclear cascade ${ }^{1-5}$ and/or the total undifferentiated hadron flux yield as a function of angle $e^{5-8}$.

B. Brief Description of the Experiment and Data Sought

The basic experimental setup is an array of steel plates separated by relatively narrow air gaps where different types of detectors can be positioned.

It is proposed that this setup also serve (perhaps slightly modified) as the beam dump for the hadron beam in Enclosure 100 of the neutrino $1 \mathrm{ab}$ or as the beam dump in the diffracted proton beam or as the beam dump in the proton laboratory. This concept has been discussed with Experimental Facilities who will also collaborate on its detailed design.

A preliminary sketch of the beam dump is shown in Fig. 1 . A re-entry cavity is not shown. It would be approximately 12 to 24 inches deep.

The information sought in this experiment includes the spatial distribution of

1. density of energy deposition;

2. radioactivation of steel, concrete and of selected soil and water specimens; 
3. hadron cascade development;

4. undifferentiated total hadron yields at large angles;

5. muon production and attenuation characteristics.

C. Beam Dump Geometry

The 50 to $500 \mathrm{GeV}$ proton beam impinges normally on the array of steel plates. These plates of graded thickness (see Table I) are separated by narrow air gaps. The various detectors may be positioned in these gaps with good precision using simple metal "hangers".

The dimensions of the plates are approximately $4^{\prime} \times 5^{\prime}$. The total length of the steel is about 10'. The exact dimensions will be a compromise between cost, remanent exposure rates at the surface, exact location of the dump, etc. The choice of lateral dimensions allows the beam dump to rest directly on the floor.

The thicknesses of the plates are graded to permit a more detailed study of the hadronic cascade at small depths where variations with depth are largest. Based on data obtained at lower energy and on the basic units of length for this problem (mean free path for high energy protons in steel $\simeq 7 "$, radiation length for steel $\simeq 0.7 "$ ), a reasonable 
choice would be ten $2^{\prime \prime}$ thick plates, followed by ten 4 " plates and the rest $6^{\prime \prime}-8 "$ plates.

For economy in construction the beam may impinge on the dump closer to a corner than the center. This exploits the cylindrical symmetry of the cascade, the large decrease in flux with radius near the beam axis and the fact that to first approximation the radial development is radially outward.

From experimental results at lower energy (Fig. 2) and from Monte Carlo calculations at higher energies (Fig. 3), it appears that measurements will become difficult at radii exceeding 45". This indicates that the proposed minimum lateral size (4' x $^{\prime}$ ') is adequate. Steel has been chosen over other material because of cost and space considerations, homogeneity, density and its essentially single element composition.

D. Detectors

D.1. Dosimeters

Two very different aspects of radiation dosimetry will be studied: (1) heating of the dump and (2) dose absorbed by organic materials.

The first problem is related to target and dump heating and resulting thermal stresses. The proposed dosimeter is a small especially designed calorimeter. 
For the second problem appropriate dosimeters are the chemical types, e.g., hydrogen-from-polyethylene, Fricke, etc.

For future reference these chemical dosimeters should be checked versus more convenient and more sensitive detectors, e.g., thermoluminescent, glass dosimeters and plastic scintillators with photomultipliers.

A summary of dosimeters is given in Table II.

D.2. Radioactivation and Remanent Exposure Rates

Samples of representative soils, low conductivity water in iron and in copper containers, aluminum, copper, steel alloys, etc. will be irradiated at various locations in the dump and their gross remanent activity will be measured.

\section{D.3. Foil Activation Detectors}

The production of various radionuclides in these foils will be measured by suitable counting equipment after irradiation. These production rates relate to particle spectra at each location. A tremendous variety of target-product combinations is possible. Our choice reflects the following considerations.

(a) The difference in excitation functions (especially in threshold) of various reactions can be exploited by combining several measurements at the same location to obtain directly crude hadron energy spectra and to make comparisons with calculations more systematic. 
(b) The times needed to count large numbers of samples vis à vis decay times of the species indicate an optimum decay time of a few hours.

(c) Direct foil counting is preferred since chemical separations become impractical for many samples on a short time scale.

(d) Production cross sections should be as large as possible and well known. Also analysis is simplified if the excitation function rises steeply beyond threshold and then flattens.

(e) Availability of foils, counting equipment, etc.

Based on these considerations, Table III contains a reasonable program for the shorter lived nuclides and corresponding targets.

In connection with Table III one can use Fig. 3 to obtain disintegration rates per unit area as predicted by the Monte Carlo calculations for different target locations.

The Al foils will be counted with a coincidence unit of two large NaI crystals. $F^{18}(2 \times 0.511 \mathrm{MeV} \gamma)$ and $\mathrm{Na}^{24}(1.37$ and $2.75 \mathrm{MeV} \gamma$ ) can be counted at the same time. $\mathrm{Na}^{22}$ may be observed in very active samples but will generally require 
longer counting times. Depending on location $1-5 \mathrm{~min}$. per foil is sufficient, giving a total counting time of $\sim 6$ hrs.

The Cu foils will be presented to a (Ge-Li) crystal and a multichannel analyzer for about $10 \mathrm{~min}$. each after $\sim 1$ day cooling. This procedure may be repeated several times in the weeks and months to follow to help identification (by decaytime considerations). Automatic data handling may be necessary.

The $\mathrm{Tb}^{149}(3.95 \mathrm{MeV} \alpha)$ from $\mathrm{Au}$ will be measured using a large diameter ( 1-1/2 inch) solid state detector with a special device insuring small detector-sample distance or a windowless gas flow proportional counter. An energy-analysis of the $\alpha$ 's counted may aid in interpretation. Limiting factors on total counting time are likely determined by the $\mathrm{Tb}^{149}$ halflife, i.e., some 4 or 5 half-lifes ( 20 hrs.) of counting time. The Au samples should also be counted on a (Ge-Li)-crystal, although the spectra may be too complex to identify all but a few conspicuous $\gamma$ quanta.

At each gap a large sheet of $\mathrm{Cu}$ (to be used as a target) will be attached to the back of the steel plate; the other foils are in turn attached to the copper sheet. They will be separated by polyethylene to prevent cross contamination. (The polyethylene may be counted to assess this problem.) After irradiation 
autoradiographs will be taken of each foilstack to find the beam center. The samples representing different radial locations can then be traced and cut. At large longitudinal distances $\mathrm{X}$-ray or Polaroid film may be added to the stack for direct irradiation.

For each kind of target foil except indium a similar detector will be present to monitor the incident beam well upstream from the beamstop. This will permit easy normalization of the data without detailed information on total number and distribution in time of incident protons or of counting efficiencies. Likewise a plastic scintillator should monitor the beam.

When indium foils will be employed it might be necessary to cover the entire dump with cadmium sheet to prevent thermal neutrons from entering the dump.

E. Hadron Yields at Large Angles

The total hadron yield in p-nucleus collisions is very important in shielding design. The various production models and empirical formulae often disagree widely, particularly at large angles. This is potentially a large source of error in shielding calculations. A measurement of the total hadron yield can be made by means of foil activation at various polar angles using the arrangement sketched in Fig. 4. 
Several different targets (Be, $\mathrm{Al}, \mathrm{Fe}, \mathrm{Cu}, \mathrm{Pb}$ ) about one-half of a mean free path long will be mounted on a low density stand along with a circular ribbon of the foils to be activated. By means of the reactions 1isted in Table III a crude, undifferentiated hadron energy spectrum can be obtained at each angle.

\section{F. Beam Profile Analyzer}

Often past experiments on beam dumps have been beset by difficulties in data interpretation at small radii. This stemmed from lack of information on the spatial distribution of the incident protons.

This distribution may be measured with a beam profile analyzer which consists of a multilayered sandwich of steel (or other material) sheets stacked parallel to the beam direction. Foils of some of the materials listed in Table III may be interleaved with the sheets. Figure 5 gives a rendition of this idea.

In addition to the spatial distribution of the beam, this stack permits a detailed study of the early development of the cascade.

Since relatively high activities may be achieved in short irradiations, this profile analyzer may be irradiated separately 
and longer lived radionuclides (e.g., $\mathrm{Mn}^{52}, \mathrm{Na}^{24}$, etc. from $\mathrm{Cu})$ are well suited. The counting of these nuclides thus need not interfere with the beam dump experiment.

G. Muon Production Measurements

Muon production will be measured near the source in order to understand muon flux measurements to be made later on at large distances.

Suitable muon detectors such as cerenkov counters in coincidence or combinations of Čerenkov and scintillation counters will be used.

These measurements will be taken behind the dump to reduce the hadron background.

Effectively, these measurements will provide a check on the calculations used in muon shielding design.

H. Apparatus

Most of the necessary instrumentation will be available as part of the regular equipment of the Radiation Physics counting laboratory. The few extra devices necessary can be obtained for about $\mathrm{K} \$ 2$. 
I. Time

The total irradiation time required at each energy is about 20 hrs plus an initial 4 hrs for readying the equipment. It is preferable to have this time available in several relatively short periods (2-3 hrs, separated by about a week) to have the opportunity to optimize the data gathering.

J. Beam

A beam of diameter about $1 \mathrm{~cm}$ or less with proton intensities in the range $10^{10}-10^{12}$ is required. Proton energy: $200 \mathrm{GeV}$ and $400 \mathrm{GeV}$.

\section{K. Manpower}

The experimenters listed above (aided by technicians of the Radiation Physics Section) are sufficient. However, interested persons from the CERN $300 \mathrm{GeV}$ study group may be invited to join us in this study (at their expense) for mutual help and benefit. 
REFERENCES

1 - A. R. Smith, et al., UCRL-11331 (Sept. 18, 1964).

2 - A. Citrón, et al., Nucl. Instr. \& Meth. 32, 48 (1965).

3 - K. Goebel \& J. Ranft, CERN 70-16 (May 27, 1970).

4 - G. Bennett, et al., to be published.

5 - F. Hajnal, et al., Nucl. Instr. \& Meth. 69, 245 (1969).

6 - M. Awschalom \& W. Schimmerling, Il Nuovo Cimento LXIV A $\underline{4}, 871$ (1969).

7 - M. Awschalom, et al., Nucl. Instr. \& Meth., 75, 93 (1969).

8 - G. S. Levine, et al., RHEL-300 GeV/RAD. NOTE/70-22 (July 8, 1970). 


\section{TABLE I}

Dump Geometry

No. of Plates
$\begin{gathered}10 \\ 10 \\ 10\end{gathered}$

Plate Thickness
2 inches
4 inches
$6-8$ inches

TABLE II

Dosimeters

\begin{tabular}{lc}
\multicolumn{1}{c}{ Type } & Range (in rads) \\
Hydrogen & $10^{5}-10^{8}$ \\
Fricke & $4 \times 10^{3}-5 \times 10^{4}$ \\
Glass & $5 \times 10^{3}-2 \times 10^{6}$ \\
Calorimeter & $3^{\circ} \mathrm{C} /$ megarad \\
Thermoluminescent & $10^{-2}-10^{6}$
\end{tabular}

TABLE III

Foil Activation Detectors

\begin{tabular}{|c|c|c|c|c|c|}
\hline Foil & Thickness & Product & $t_{12}$ & Threshold & Sensitivity* \\
\hline A1 & $20 \mathrm{mil}$ & $F^{18}$ & $110 \mathrm{~m}$ & $45 \mathrm{MeV}$ & $2 \times 10^{6}$ \\
\hline $\begin{array}{c}C \text { (polyeth- } \\
\text { ylene) }\end{array}$ & $2 \mathrm{mil}$ & $\mathrm{C}^{11}$ & $20 \mathrm{~m}$ & $20 \mathrm{MeV}$ & $4 \times 10^{8}$ \\
\hline A1 & $20 \mathrm{mil}$ & $\mathrm{Na}^{24}$ & $15 \mathrm{~h}$ & $6 \mathrm{MeV}$ & $6 \times 10^{5}$ \\
\hline$S$ & $\sim 1 / 8$ inch & $\mathrm{P}^{32}$ & $14.3 \mathrm{~d}$ & $2.3 \mathrm{MeV}$ & $2 \times 10^{4}$ \\
\hline $\mathrm{Cu}$ & $1 \mathrm{~mm}$ & $\mathrm{Mn}^{52}, \mathrm{Na}^{24}$ & $5.6 \mathrm{~d}$ & $100 \mathrm{MeV}$ & $2 \times 10^{5}$ \\
\hline In & $20 \mathrm{mil}$ & $\operatorname{In}^{1} 16 \mathrm{~m}$ & $54 \mathrm{~m}$ & thermal & -- \\
\hline $\mathrm{Au}$ & $0.5 \mathrm{mil}$ & $\mathrm{Tb}^{149}$ & $4.1 \mathrm{~h}$ & $600 \mathrm{MeV}$ & $2 \times 10^{1}$ \\
\hline
\end{tabular}

$\bar{*}$ dis/sec. at end of $6 \mathrm{~h}$ irradiation of beam monitor (for $10^{11}$ prot./sec.) 


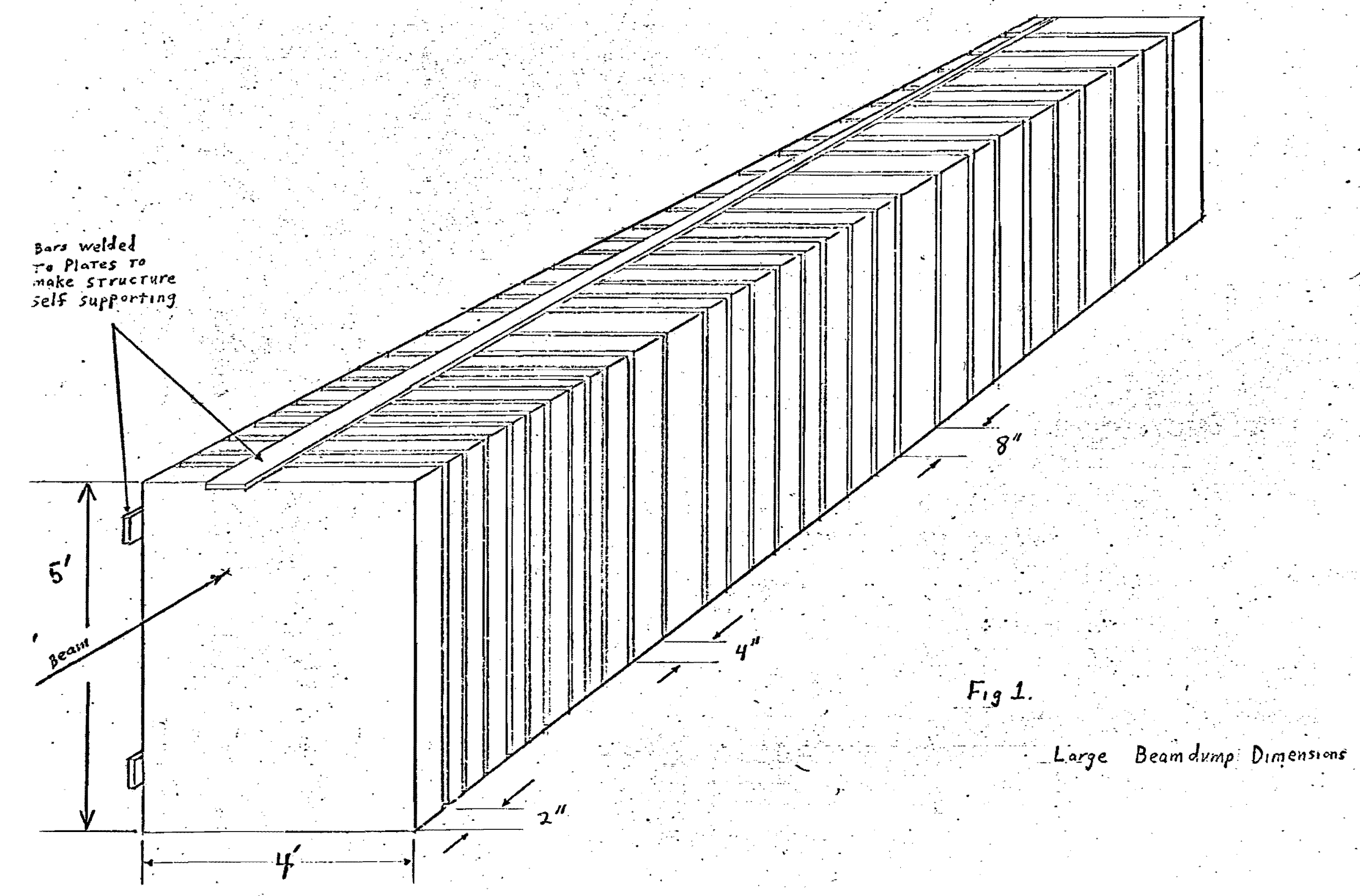




\section{Fig 2 \\ $\frac{\text { \#ptcls }(T>45 \mathrm{Mer})}{\mathrm{cm}^{2} \text { inc.proton based on } F^{18} \text { from } A l \text {; isocontours }}$}

for 29.4 Geu $P$ on $F_{C}$

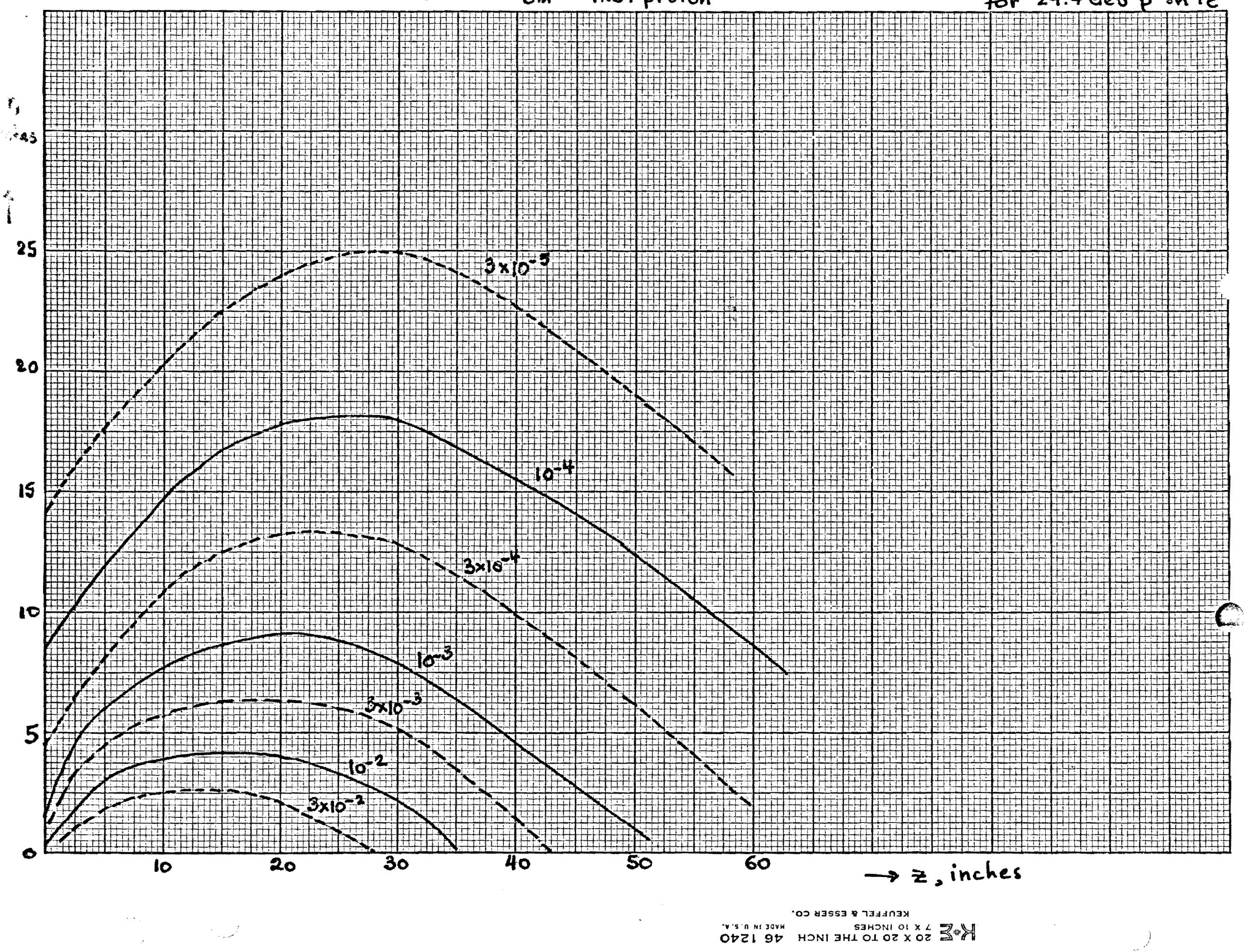




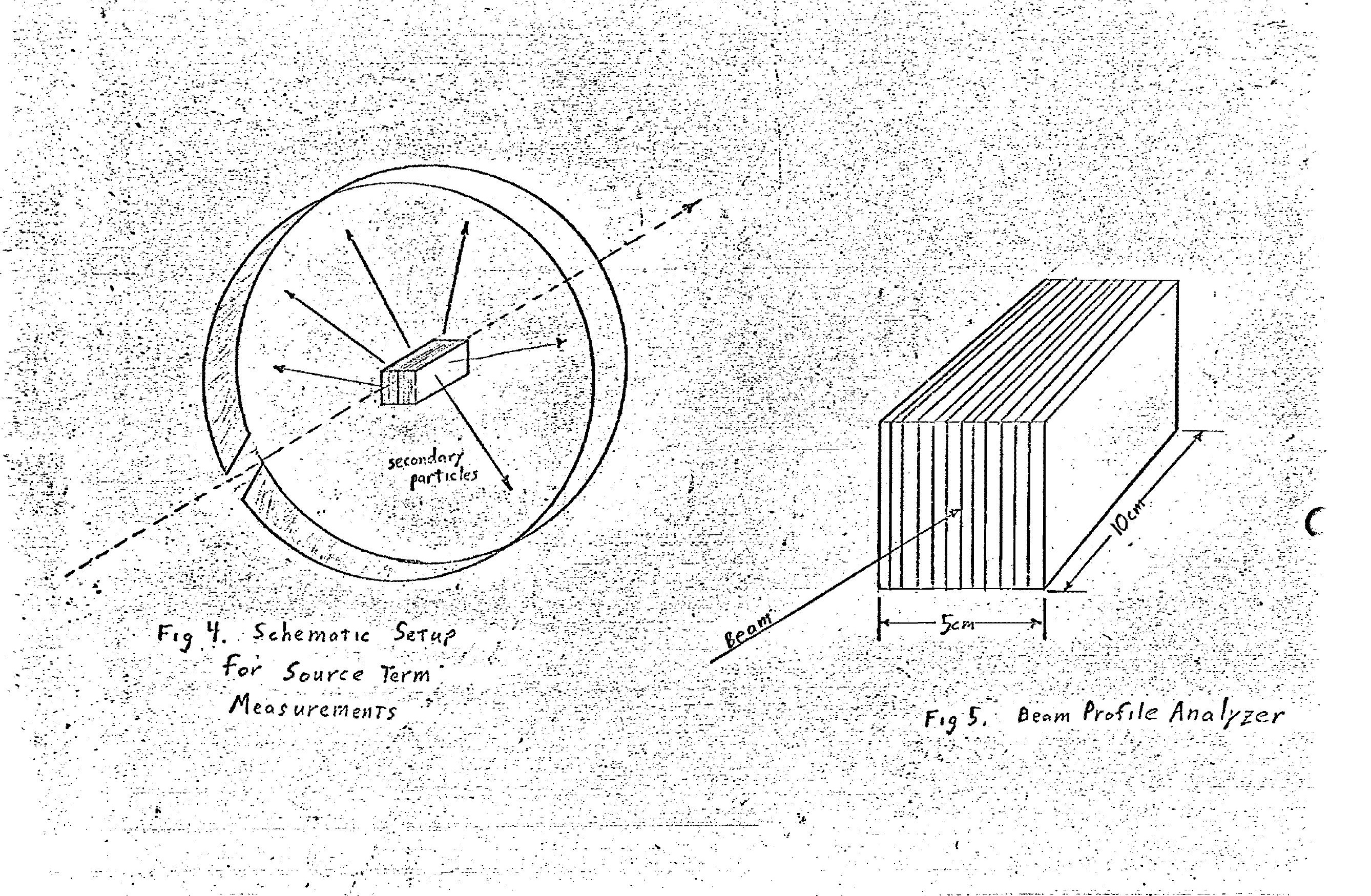

